\title{
ESTUDO DA REAÇÃO CICATRICIAL E DA RESPOSTA À VENTILAÇÃO MECÂNICA INTRAOPERATÓRIA EM TRANSPLANTES SEGMENTARES DE TRAQUÉIA - AUTÓLOGOS E HOMÓLOGOS - EM COELHOS
}

\author{
Cicatricial behavior and response to intraoperatory mechanical ventilation of the transplanted \\ segment of the trachea (autologous and homologous transplant of the entire segment) in rabbits
}

Roberto Salvador de Souza Guimarães, João Batista Vieira de Carvalho, João Vidal de Carvalho Polido Lopes, Gustavo Marques de Oliveira

\begin{abstract}
RESUMO
Objetivo: avaliar o comportamento cicatricial e a resposta à ventilação mecânica intra-operatória do segmento transplantado da traquéia em coelhos. Métodos: foram testados dois grupos: transplante autólogo (grupo I) e transplante homólogo (grupo II) segmentar total da traquéia de coelhos. Resultado: Não houve diferença estatística significativa pelo teste de Qui-quadrado $(\mathrm{p}<0,05)$ com relação à variação de peso entre os grupos estudados. Com relação ao tempo de sobrevida pelo teste de Qui-quadrado ( $\mathrm{p}<0,05)$, as médias foram consideradas estatisticamente diferentes entre si $(\mathrm{tc}=-3,60)$, com a sobrevida apresentada pelos animais do grupo I maior que a sobrevida apresentada pelos animais do grupo II. Na necroscopia direta e avaliação radiológica, observou-se pelo teste Kruskal-Wallis (p<0,05) que não houve diferença estatística significativa entre os grupos estudados. Observaram-se complicações como estenose, formação de granuloma e fístulas em ambos os grupos. Com relação ao comportamento intra-operatório e à resposta à ventilação mecânica pelo teste de KruskalWallis $(\mathrm{p}<0,05)$, não se observou diferença estatística significativa entre os Grupos I e II. Tanto os animais do Grupo I como os do Grupo II apresentaram boa resistência à hiperpressão da ventilação mecânica intra-operatória. Com relação aos resultados anatomopatológicos pelo teste de Kruscal-Wallis $(\mathrm{p}<0,05)$, não se observou diferença estatística significativa entre os Grupos I e II. Nos animais do Grupo I, observou-se maior prevalência de granulomas, metaplasia óssea, necrose na região da anastomose, fibroplasia, reepitelização do enxerto, infiltrado polimorfonuclear. Conclusão: foi possível observar uma reepitelização e processos inflamatórios tanto precoces quanto tardios nos grupos. Os animais com transplante autólogo apresentaram melhor evolução clínica com sobrevida maior que aqueles que receberam transplante homólogo.
\end{abstract}

Descritores: Traquéia, Coelhos, Transplante, Ventilação Mecânica, Evolução Clínica, Sobrevida.

\section{Instituição:}

Departamento de Cirurgia Experimental - Faculdade de Ciências Médicas - Unifenas - MG Universidade José do Rosário Vellano - Alfenas - MG

\section{Correspondência:}

Roberto Salvador de Souza Guimarães

Alameda Libânio, 460 - Jardim da Colina

CEP 37130-000 - Alfenas - MG

Tel./ Fax: 5535 3291-6248

E-mail: rodanvic27@gmail.com

Recebido em: 05.03.2007

\section{INTRODUÇÃO}

As doenças que acometem a traquéia, traumáticas ou não, têm aumentado de freqüência nos últimos anos. ${ }^{1-4}$ A assistência ventilatória prolongada e a cirurgia curativa do câncer podem determinar destruições traqueais extensas, de difícil correção cirúrgica. ${ }^{5,6}$ As complicações como estenoses e fístulas, também são de difícil correção e envolvem refinamentos de técnica cirúrgica. A evolução pós-operatória depende da resposta à ventilação mecânica pós-operatória e da cicatrização. Com os avanços da cirurgia moderna e dos aparelhos de suporte ventilatório, o reparo da traquéia deve manter a via respiratória prévia por meio de paredes resistentes e promover o crescimento do próprio epitélio traqueal. ${ }^{7-9}$

Sempre que a anatomia da traquéia permitir, deve-se tentar reconstruí-la com seu próprio tecido. A dissecção cuidadosa do tecido conjuntivo e da fáscia cervical interna permite, geralmente, mobilização suficiente da traquéia para seu reparo primário. 
O melhor restabelecimento é por meio da anastomose términoterminal dos cotos. A reconstrução direta permite manter a continuidade da mucosa com seu aparelho ciliar, que é fundamental para a mobilização do muco e eliminação de corpos estranhos. ${ }^{10-12}$

Foi realizada em cães, com sucesso, a primeira transecção traqueal com anastomose término-terminal ${ }^{13}$ Foram obtidos bons resultados com a reconstrução primária término-terminal em humanos com estenose de traquéia. ${ }^{13,14}$

Têm sido relatados casos de reparo de lesões traqueais com emprego do transplante de pele, sendo esses estudos precursores de uma era importante da terapêutica cirúrgica. ${ }^{15}$ Os princípios básicos da reconstrução traqueal consistem em utilizar, sempre que possível, tecidos autólogos e evitar tensão da sutura. A reconstrução plástica de traquéia foi também relatada ${ }^{16}$ para cobrir um defeito anterior da traquéia com enxerto pediculado de pele e da cartilagem costal. Foram ressecados 10 anéis traqueais em um paciente com câncer de tireóide que invadia a traquéia, e, pela primeira vez, esse segmento foi substituído por uma prótese de material não especificado, com obtenção de uma sobrevida de cinco anos. ${ }^{17}$

Bons resultados com cirurgia traqueal têm ocorrido no passar dos anos. O surgimento de próteses e aparelhos respiratórios sofisticados estimula-nos a novas pesquisas. ${ }^{18,19}$ Os novos avanços culminaram com a realização do primeiro transplante de traquéia em humano. ${ }^{20}$

Em várias instituições de saúde tem se tornado comum a confecção de traqueotomias após cirurgias de grande porte e em pacientes graves não cirúrgicos que requerem internação prolongada em unidades de tratamento intensivo. Há doentes que se recuperam e se sentem incomodados com as traqueotomias definitivas, causadoras de sofrimento.

Os trabalhos na literatura evidenciaram que foi desenvolvido um grande número de pesquisas, com o intuito de encontrar um substituto ideal para a traquéia. No passado, a estenose da traquéia era tratada de forma conservadora, com sobrevida apenas dos pacientes, e, particularmente, crianças com estenose congênita que apresentavam melhoria com o crescimento. ${ }^{21,22} \mathrm{Na}$ década de 1980 , apesar da introdução de novas práticas cirúrgicas, a mortalidade referida continuava elevada, variando entre 7 e $50 \%$ de acordo com os centros, com taxa de reoperação de $22 \%{ }^{21,23}$ A utilização de circulação extra-corpórea permitiu melhores resultados cirúrgicos no tratamento de estenose em crianças, com uma taxa de sucesso significativa em alguns centros. As técnicas atualmente utilizadas são anastomose término-terminal, traqueoplastia de alargamento e traqueoplastia de deslizamento. A anastomose término-terminal somente é indicada quando a estenose não excede $50 \%$ do comprimento da traquéia, pois resulta em encurtamento significativo. ${ }^{1,24}$ A traqueoplastia de alargamento com colocação de pericárdio ou cartilagem heterólogos tem complicações freqüentes, como a formação de tecido de granulação endoluminal, estenose e mediastinite, e necessita de longos períodos de intubação póscirúrgica. ${ }^{2,3}$ Os índices de sobrevivência são de $33 \%$ nas primeiras séries e $83 \%$ nas mais recentes, com necessidade de reoperação em $21-33 \%$ dos casos. ${ }^{6}$ A utilização de auto-enxerto de traquéia pareceu melhorar o prognóstico, com mortalidade operatória de $10 \%$ e redução das complicações. ${ }^{8}$

A traqueoplastia de deslizamento ${ }^{25}$ consiste na secção transversal da traquéia no ponto médio da estenose, com a extremidade proximal mobilizada posteriormente e depois seccionada longitudinalmente ao longo de todo o comprimento da parede anterior da estenose, abaixo da cricóide. A extremidade distal é mobilizada anteriormente e depois dividida longitudinalmente ao longo da parede posterior da estenose até a porção membranosa acima da carina e os dois segmentos da traquéia são aproximados com deslizamento de um sobre o outro. A dissecção lateral é evitada, para assegurar um bom suprimento vascular. Obtém-se assim a duplicação do perímetro intra-luminal e o quádruplo do diâmetro da estenose. Parece ser, para alguns autores, a melhor opção. Comporta menos riscos, embora semelhantes aos referidos para a traqueoplastia de alargamento, encurtando a traquéia em metade do comprimento da estenose; a anastomose oblíqua e longa permite menor tensão nas suturas, e a dissecção limitada preserva o fornecimento sanguíneo, reduzindo a isquemia. A estabilidade obtida pela técnica permite a extubação precoce. Por outro lado, nenhum dos sobreviventes apresentou limitações respiratórias com necessidade de colocação de prótese endoluminal ou traqueotomia, como aconteceu em 9-60\% de casos, de acordo com diferentes séries, na operação de traqueoplastia de alargamento. A taxa de sobrevivência foi de 73-82\%, e foi maior nas situações de estenose traqueal isolada do que nos casos acompanhados de outras malformações, sobretudo malformações cardiovasculares graves. Nos casos em que coexistiam malformações graves em crianças com estenose traqueal congênita, verificou-se melhoria prognóstica quando foi possível a correção simultânea das malformações por meio de intervenção cuidadosamente planejada por equipe multidisciplinar. ${ }^{25,26}$

Dentro da linha de pesquisa relativa à reconstrução traqueal, e por ser assunto atual e de relevante interesse, decidiu-se realizar estudo para observar o comportamento cicatricial e de resposta à ventilação mecânica do auto-transplante e transplante homólogo de traquéia em coelhos.

Apesar das experiências feitas em diversos trabalhos nos últimos cinqüenta anos, nenhuma prótese ou enxerto serviu de molde para o crescimento da parede cartilaginosa da traquéia ou de seu epitélio interno. Isso demonstra a necessidade de mais pesquisas para se obter procedimentos mais seguros na reconstrução de segmentos danificados da traquéia.

\section{MATERIAL E MÉTODO}

O estudo foi realizado no laboratório de cirurgia experimental da Universidade José do Rosário Vellano, localizada no campus da cidade de Alfenas (MG). Foram estudados 20 (vinte) coelhos adultos de ambos os sexos, da raça Nova Zelândia, aparentemente sadios e com peso médio de 3500 - 4500 gramas, obtidos do Biotério da própria Instituição. Foram distribuídos de forma aleatória em dois grupos, de acordo com a técnica cirúrgica empregada:

Grupo I - Animais submetidos ao transplante autólogo de segmento circunferencial total de traquéia $(n=10)$;

Grupo II - Animais submetidos ao transplante homólogo de segmento circunferencial total de traquéia $(\mathrm{n}=10)$.

A técnica anestésica ${ }^{10}$ utilizada compreendeu os seguintes períodos:

- Pré-anestésico: Intervalo de tempo compreendido entre a indicação anestésica e o início da anestesia, após administração das medicações pré-anestésica (Atropina 0,014 mg. $\mathrm{Kg}^{-1} \mathrm{e}$ Midazolam 0,2 mg. $\mathrm{Kg}^{-1}$ ).

- Trans-anestésico: Intervalo entre o início da anestesia até o início da recuperação. Com cuidados dirigidos para a verificação dos 
reflexos pertinentes ao plano anestésico desejado, observação da posição adequada do animal, principalmente da cabeça, evitando pressões sobre a curvatura da sonda endotraqueal.

- Pós-anestésico: Intervalo de tempo entre o início da recuperação e o restabelecimento total da consciência e parâmetros fisiológicos.

Após os períodos descritos anteriormente, iniciou-se a técnica de associação anestésica, ou seja, técnica que permitiu intervenção mais prolongada. Utilizou-se a associação de Cloridrato de Cetamina e Cloridrato de Xilazina na dosagem de 0,1 mL. 200 gramas de peso vivo $\left(0,5 \mathrm{~mL} \cdot \mathrm{kg}^{-1}\right)$ intramuscular profunda em partes iguais da mistura. Esperada a latência dos anestésicos, procedeu-se o posicionamento dos animais na goteira de Claude Bernard, e realizou-se:

1. Ventilação manual sob máscara com sistema de Mapleson D, até o restabelecimento da respiração espontânea, em que não houve intubação orotraqueal;

2. Tricotomia da região cervical;

3. Instalação do estetoscópio precordial;

4. Antissepsia da pele com PVPI tópico;

5. Infiltração com Cloridrato de Lidocaína $2 \%$ sem vasoconstritor em região mediana cervical;

6. Colocação campos primários e secundários;

7. Dissecção e cateterização de veia jugular direita;

8. Infusão de solução fisiológica $0,9 \%\left(2 \mathrm{~mL} \cdot \mathrm{kg}^{-1} \cdot \mathrm{h}^{-1}\right)$;

$\mathrm{O}$ ato cirúrgico, em condições estéreis, teve início com incisão mediana cervical anterior, de aproximadamente $10 \mathrm{~cm}$ de extensão da cartilagem tireóide, a $2 \mathrm{~cm}$ da fúrcula esternal, interessando a pele e o tecido celular subcutâneo; a seguir, após dissecção do plano muscular, a traquéia foi completamente exposta. Delimitada a área traqueal a ser ressecada, foi retirado fragmento do enxerto de traquéia, compreendendo de três a cinco anéis traqueais, no terço médio da traquéia cervical. A seguir, foram passados pontos em "U" com náilon monofilamentar 6-0 nos quatros ângulos entre os cotos de traquéia e o enxerto. Esses pontos foram utilizados para fixar novamente o segmento retirado (auto-transplante total) ou o correspondente de traquéia, homólogo, das mesmas dimensões, obtido de outro coelho com a mesma técnica descrita (transplante homólogo total) por meio de sutura contínua com náilon 6-0 monofilamentar. Esses pontos foram passados ao longo de toda borda do retalho traqueal, de forma a permitir a aposição perfeita do mesmo sobre a traquéia. Para se obter o ajuste perfeito, foi necessário que cada ponto fosse passado somente por um anel traqueal. Antes do término da sutura, foi promovida a passagem da cânula de intubação de forma retrógrada.

Ao término da sutura, a consistência da anastomose foi testada, deixando-se a mesma imersa em solução fisiológica e promovendo hipertensão na cânula de intubação, colocada proximalmente à anastomose. Utilizou o sistema de Bain, Takaoka 3312.

Nos casos de vazamento de ar visualizado pela formação de bolhas, foram dados pontos adicionais e adicionadas gotas de Éster de Cianoacrilato em região anterior, até cessar o vazamento por completo. A reconstrução cirúrgica foi feita por planos anatômicos e realizado curativo simples, limpeza da ferida cirúrgica com solução fisiológica $0,9 \%$ e PVPI tópico, com colocação de faixa protetora.
No período pós-operatório imediato, aguardou-se o despertar dos animais e o retorno da respiração espontânea para extubação. Em todos os animais foi administrada dose única de Tilosina (1mL) como antibioticoprofilaxia por via intramuscular e os coelhos permaneceram em gaiolas separadas.

No transplante homólogo total, um segmento total de traquéia compreendendo três anéis foi retirado e transplantado para outro animal em tempos simultâneos. A técnica anestésica e cirúrgica utilizada foi a mesma descrita anteriormente.

Todos os coelhos foram observados diariamente quanto ao aspecto geral, interesse pelo ambiente, apetite, alimentação, sede, evacuação, tosse, estudos respiratórios, retrações, tiragem, cornagem e condições locais de cicatrização.

Foram realizadas radiografias das regiões cervical e torácica em todos os coelhos no dia posterior à cirurgia, em posição de decúbito lateral sob sedação. $\mathrm{O}$ objetivo foi constatar a perviedade da luz da traquéia e o extravasamento de ar para os tecidos paratraqueais através de soluções de continuidade nas anastomoses porventura existentes.

Macroscopicamente foram avaliados a traquéia, os enxertos e os locais de sutura, para verificar as alterações, presença de secreções, deformidades, tecido de granulação e deiscência.

Procedeu-se a ressecção da traquéia transplantada juntamente com um segmento proximal e um segmento distal aparentemente normais da traquéia nos coelhos que evoluíram para óbito ou foram sacrificados por ocasião do $30^{\circ}$ dia pós-operatório, para estudo anatomopatológico. Foram imersos em solução de formol a $10 \%$. Os segmentos fixados foram recortados com lâmina de bisturi, com o cuidado de conservar todas as camadas de tecido. A desidratação desses segmentos foi efetuada em bateria de álcool e xilol. Após diafanização no xilol, os segmentos foram incluídos a vácuo em parafina e, em seguida, recortados pelo micrótomo. Após reidratação em bateria de xilol e álcool, os cortes foram corados com hematoxilina-eosina para verificação do comportamento cicatricial, rejeição, processo inflamatório e proliferação granulomatosa.

A peça foi encaminhada para estudo histopatológico, incluída em parafina e coloração de hematoxilina e eosina no laboratório de histopatologia da Unifenas. Foi estudada a cicatrização e a incorporação do enxerto traqueal à traquéia dos animais por meio das evidências de rejeição e deiscência, com especial atenção às alterações relacionadas com a absorção do enxerto, substituição por fibrose, presença de necrose na região da anastomose, rejeição, crescimento de tecido ósseo ou cartilaginoso, granulomas, estenoses, metaplasia óssea, fibroplasia, crescimento de epitélio interno, infecção, proliferação de colágeno, neoformação vascular, proliferação de colágeno e presença de microfístulas.

Na avaliação da rejeição atribuiu-se o grau de rejeição aos enxertos baseando-se nos parâmetros:

Grau 0-ausente: ausência de infiltrado linfocitário.

Grau 1-leve: presença de processo infiltrativo de linfócitos de forma escassa na anastomose;

Grau 2-moderado: presença de processo infiltrativo linfocitário na anastomose e ao longo do enxerto;

Grau 3-intenso: presença de grande número de células, caracterizando processo infiltrativo linfocitário intenso ao nível da anastomose e ao longo do enxerto; 
Grau 4-muito intenso (rejeição): destruição da arquitetura do enxerto, com subversão, reabsorção do mesmo associado a estenose, tecido granulomatoso e/ou microfístulas.

Para verificar a heterogeneidade entre os grupos, utilizou-se o teste Mann-Whitney. Para avaliar a distribuição do sexo, peso e a sobrevida dos animais entre os grupos, utilizou-se o teste do Qui-quadrado.

A resposta à ventilação mecânica, cicatricial e o grau de rejeição entre os grupos foram comparados pelo teste de Kruskall-Wallis com avaliação das médias pelo teste $\mathrm{t}$-Student. Foi adotado o nível de significância de $\mathrm{p}<0,05$.

\section{RESULTADOS}

Quanto à distribuição por sexo, os grupos mostraram-se homogêneos, com pequena variação no Grupo II, com prevalência do sexo masculino (Tabela 1).

Não houve diferença significativa pelo teste de Qui-quadrado $(p<0,05)$ com relação ao peso entre os grupos estudados. Observouse, porém, maior perda de peso no Grupo II (Tabela 1).

Com relação à sobrevida dos grupos I e II pelo teste de Quiquadrado $(\mathrm{p}<0,05)$, as médias foram consideradas estatisticamente diferentes entre si $\left(t_{c}=-3,60\right)$, com a sobrevida apresentada pelos animais do Grupo I maior que a sobrevida apresentada pelos animais do Grupo II (Tabela 1).

Com relação à avaliação clínica diária pelo teste de Qui-quadrado $(p<0,05)$, não se observou diferença estatística significativa com relação aos parâmetros avaliados entre os grupos estudados. Os animais do Grupo II evoluíram com complicações associadas a maiores variações de peso com perda ponderal.

Na necroscopia direta e na avaliação radiológica observou-se pelo teste Kruskal-Wallis $(\mathrm{p}<0,05)$ que não houve diferença estatística significativa entre os grupos com relação à ocorrência de complicações como estenose, fístulas, enfisema subcutâneo, pneumomediastino e infecção (Tabela 2) e (Figura 2 e 3).

Tabela 1: Distribuição nos Grupos I (transplante autólogo) e II (transplante homólogo) por peso inicial e final (gramas), sobrevida (dias) e variação de peso (gramas).

\begin{tabular}{ccccc}
\hline & \multicolumn{2}{c}{ Peso inicial $\mathbf{( g )}$} & \multicolumn{2}{c}{ Peso final $(\mathbf{g})$} \\
\hline Grupo I & Grupo II & Grupo I & Grupo II \\
\hline Média \pm DP & $4055 \pm 107,93$ & $4046 \pm 79,89$ & $4099 \pm 80,48$ & $4041 \pm 71,87$ \\
Valor máximo & 4250 & 4180 & 4200 & 4160 \\
Valor mínimo & 3920 & 3950 & 3980 & 3900 \\
\hline & Sobrevida (dias) & Variação de peso (g) \\
\hline \multirow{2}{*}{ Média \pm DP } & $33,90 \pm 10,77$ & $16,80 \pm 10,04$ & $-44 \pm 65,18$ & $5 \pm 38,94$ \\
Valor máximo & 51 & 29 & 50 & 60 \\
Valor mínimo & 22 & 2 & 170 & 70 \\
\hline
\end{tabular}

Teste Qui-quadrado $(p<0,05)$.
Tabela 2: Complicações macroscópicas encontradas nos animais do Grupo I e $/ 1$ e as datas das respectivas cirurgias.

\begin{tabular}{|c|c|c|c|c|c|c|}
\hline Animais & Estenose & Fístula & $\begin{array}{c}\text { Enfisema } \\
\text { Subcutâneo }\end{array}$ & $\begin{array}{l}\text { Pneumo- } \\
\text { mediastino }\end{array}$ & Infecção & $\begin{array}{l}\text { Data da } \\
\text { Cirurgia }\end{array}$ \\
\hline 1 & NÃO & NÃO & NÃO & NÃO & SIM & $01 / 04 / 2006$ \\
\hline 2 & NÃO & NÃO & SIM & NÃO & SIM & $12 / 04 / 2006$ \\
\hline 3 & NÃO & NÃO & NÃO & NÃO & NÃO & $24 / 04 / 2006$ \\
\hline 4 & NÃO & NÃO & SIM & NÃO & NÃO & $13 / 03 / 2006$ \\
\hline 5 & NÃO & NÃO & SIM & SIM & SIM & $01 / 04 / 2006$ \\
\hline 6 & NÃO & NÃO & SIM & NÃO & NÃO & 06/03/2006 \\
\hline 7 & NÃO & NÃO & NÃO & NÃO & NÃO & $11 / 03 / 2006$ \\
\hline 8 & NÃO & NÃO & NÃO & NÃO & SIM & $13 / 03 / 2006$ \\
\hline 9 & SIM & NÃO & SIM & NÃO & NÃO & $06 / 03 / 2006$ \\
\hline 10 & NÃO & NÃO & NÃO & NÃO & SIM & $10 / 03 / 2006$ \\
\hline Animais & Estenose & Fístula & $\begin{array}{c}\text { Enfisema } \\
\text { Subcutâneo }\end{array}$ & $\begin{array}{l}\text { Pneumo- } \\
\text { mediastino }\end{array}$ & Infecção & $\begin{array}{l}\text { Data da } \\
\text { Cirurgia }\end{array}$ \\
\hline 1 & SIM & NÃO & SIM & NÃO & SIM & $07 / 11 / 2006$ \\
\hline 2 & NÃO & SIM & SIM & NÃO & NÃO & $24 / 10 / 2006$ \\
\hline 3 & NÃO & NÃO & SIM & NÃO & NÃO & $07 / 11 / 2006$ \\
\hline 4 & NÃO & SIM & SIM & NÃO & SIM & $28 / 11 / 2006$ \\
\hline 5 & NÃO & NÃO & SIM & NÃO & NÃO & $20 / 02 / 2006$ \\
\hline 6 & NÃO & NÃO & SIM & NÃO & NÃO & $11 / 02 / 2006$ \\
\hline 7 & SIM & $N \tilde{A} 0$ & NÃO & NÃO & NÃO & $11 / 02 / 2006$ \\
\hline 8 & SIM & NÃO & SIM & NÃO & SIM & $28 / 11 / 2006$ \\
\hline 9 & NÃO & NÃO & SIM & NÃO & SIM & 28/11/2006 \\
\hline 10 & SIM & NÃO & NÃO & NÃO & NÃO & $12 / 02 / 2006$ \\
\hline
\end{tabular}

Com relação aos achados anatomopatológicos pelo teste KruskallWallis $(\mathrm{p}<0,05)$, não se observou diferença estatística significativa entre os Grupos I e II. Tanto nos animais do Grupo I como nos animais do Grupo II observou-se presença de granulomas, áreas de necrose na região da anastomose, fibroplasia, reepitelização do enxerto, infiltrado polimorfonuclear, focos de ulceração e reação inflamatória, que ocorreu de forma similar em ambos os grupos. A cicatrização ocorreu com freqüência similar em ambos os grupos. Também foram observados focos de ulceração do epitélio em freqüência similar nos animais dos grupos estudados (Figura 4).

A resposta à ventilação mecânica foi testada por meio da hiperpressão na cânula de intubação, para testar a consistência da anastomose e observar o surgimento de vazamentos e outros sinais de ruptura traqueal. Na pressão entre 20 e $25 \mathrm{~cm} \mathrm{H_{2 }} \mathrm{O}$ não houve ruptura completa da anastomose nos animais dos grupos estudados. Em nenhum dos animais houve necessidade de ressutura completa do enxerto traqueal. (Figura 1). 


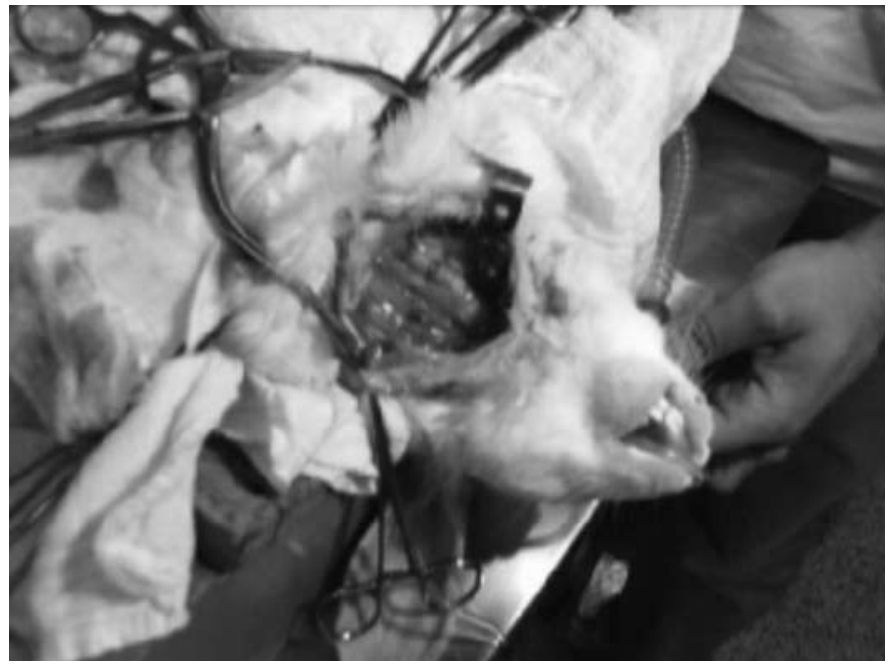

Figura 1: Término, teste e resposta da anastomose à ventilação.

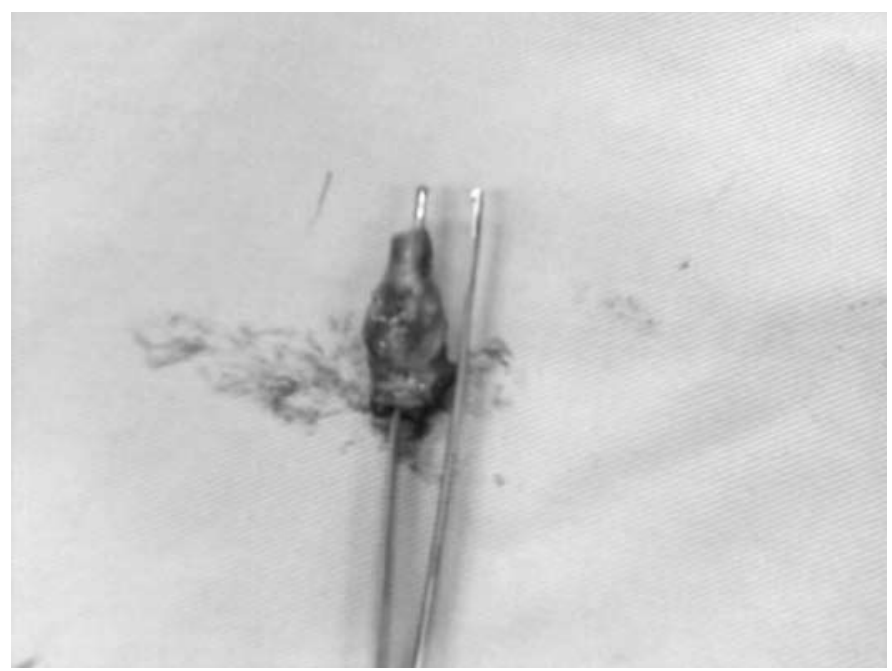

Figura 3: Peça para estudo anatomopatológico.

Com relação ao comportamento intra-operatório e à resposta à ventilação mecânica pelo teste Kruskal-Wallis $(p<0,05)$, não se observou diferença estatística significativa entre os Grupos I e II. Tanto os animais do Grupo I como os do Grupo II apresentaram boa resistência à hiperpressão da ventilação mecânica intraoperatória e pós-anastomose.

\section{DISCUSSÃO}

Observou-se que tanto o transplante autólogo como o homólogo de segmentos circunferenciais de traquéia apresentaram-se factíveis experimentalmente em coelhos com complicações e viabilidade similares. Conforme evidenciado em outros estudos, ${ }^{27-31} \mathrm{a}$ ocorrência de rejeição, infecção, estenose, fístula e outras alterações cicatriciais podem ocorrer em ambos os tipos de enxertos. O maior tempo de sobrevida observado nos animais do Grupo I evidencia maior aceitação de enxerto autólogo em relação ao homólogo.

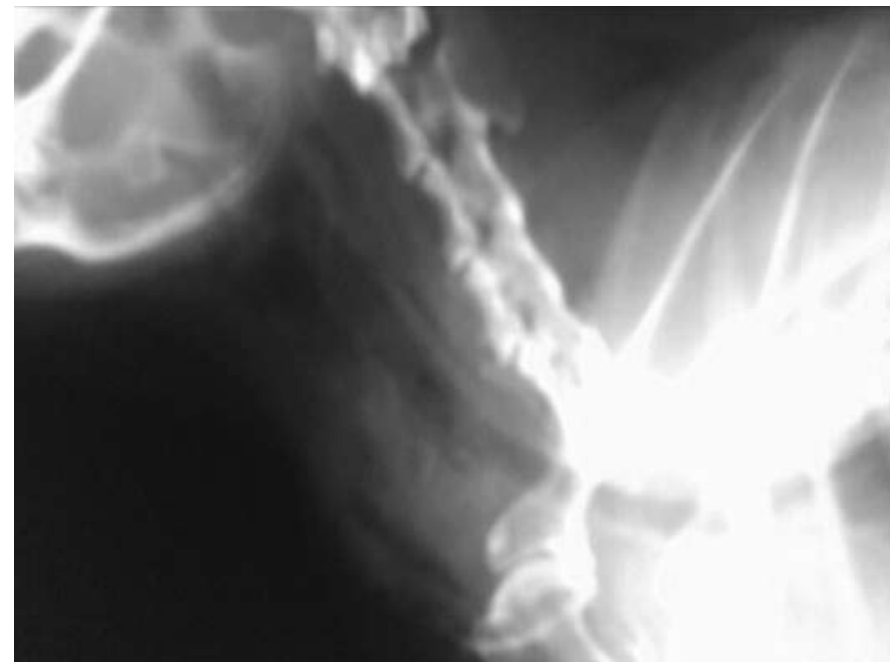

Figura 2: Estudo radiológico um dia após transplante.

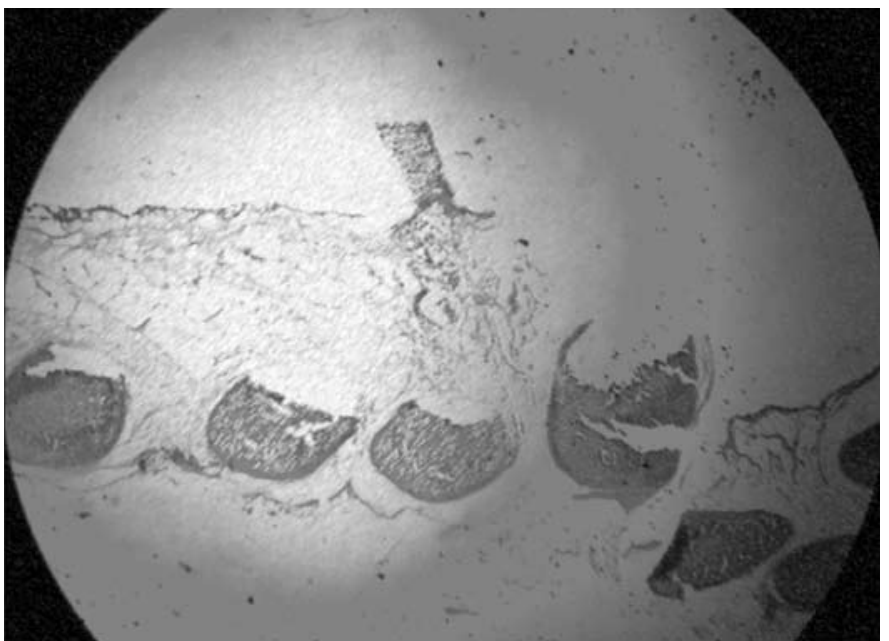

Figura 4: Corte histológico que evidencia fragmento de traquéia com extenso infiltrado linfocitário, neutrofílico, e áreas de necrose na mucosa. Presença de múltiplos focos de calcificação eucondrial. Observam-se ainda vasos neoformados, áreas de fibrose e reepitelização difusa do epitélio traqueal, com aspecto hiperplásico.

Com relação à avaliação clínica diária, os animais do Grupo II apresentaram menor estabilidade clínica pós-cirúrgica, como se observou pela repercussão sobre as variações de peso e sobrevida. Essa resposta mais favorável ao transplante autólogo tem sido relatada desde os primeiros estudos realizados em traquéia de cães, e demonstra a maior receptividade do enxerto autólogo. ${ }^{32} \mathrm{Na}$ presente pesquisa, observamos que ambos os enxertos, autólogo e homólogo, são factíveis. Embora os animais de ambos os grupos estudados tenham apresentado complicações, os animais do Grupo I mostraram-se mais estáveis no pós-operatório imediato e tardio.

Nos primeiros 23 dias pós-transplante, a ocorrência de infecção foi observada em cinco animais do Grupo I e em quatro animais do Grupo II no local do enxerto. Verificou-se processo inflamatório crônico acutizado, associado à microabscessos nas duas extremidades do enxerto, formando ulceração do epitélio de 
revestimento, com áreas de proliferação fibroblástica e presença de polimorfonucleares com cicatrização incipiente nesses casos.

Conforme observado por outros autores, ${ }^{31}$ no período de quatro a seis semanas pós-transplante houve substituição das áreas de reabsorção cartilaginosa por tecido conjuntivo denso associado a segmentos de necrose. Essas áreas mostraram-se mais frágeis com diminuição da luz traqueal, estenose e formação de microfístulas e fístulas. Alguns autores associaram essas alterações a uma possível rejeição ao tecido transplantado. . $^{33,34}$

Não utilizamos técnicas imunossupressoras para controlar ou alterar o processo inflamatório. A rejeição não parece ser um fator decisivo na aceitação tanto do enxerto transplantado autólogo como homólogo de traquéia. Esse comportamento do tecido traqueal frente à rejeição foi observado por outros autores, que não evidenciaram claramente fenômenos de rejeição e indução de reações imunitárias discretas e inespecíficas associados ao transplante autólogo e homólogo de traquéia. ${ }^{35} \mathrm{O}$ uso de imunossupressores ou a esplenectomia não alteram significativamente a evolução pós-operatória do enxerto traqueal transplantado. ${ }^{33,34}$

A cartilagem traqueal foi substituída por colágeno com formação de estenose circunferencial parcial em um animal do Grupo I e em 4 animais do Grupo II. Devido à estenose da luz traqueal, seriam de se esperar alterações respiratórias e mesmo morte dos animais. Alguns animais do Grupo II apresentaram essas complicações com estridor, cornagem e dispnéia, associadas à dificuldade de alimentação com conseqüente perda ponderal. Um animal do Grupo I apresentou essas complicações, porém não houve alteração substancial na alimentação e perda significativa de peso.

A utilização de éster de cianocrilato para vedar alguns vazamentos nas anastomoses associou-se à presença de granuloma de corpo estranho e processo inflamatório, e se revelou um bom método auxiliar para a aerostasia pós-anastomose cirúrgica. Não se observou bloqueio da migração celular relacionado à colocação do éster de cianocrilato.

Nas áreas anastomóticas não se observou presença marcante de fibras elásticas. A presença de fibras elásticas está implicada com variações no comprimento, diâmetro e posição da traquéia normal durante a respiração, deglutição e movimentos do pescoço. As fibras elásticas funcionalmente teriam a capacidade de alterar a luz traqueal, se não em sua totalidade, pelo menos em dois terços dela. Outros fatores relacionados a alterações da mobilidade traqueal seriam a formação de colágeno e calcificação em grande parte da cartilagem do enxerto transplantado. Essas alterações foram observadas em ambos os grupos, e podem ter influenciado a evolução pós-operatória em parte dos animais estudados.

A ocorrência de fístulas decorre de fenômenos isquêmicos, necrose nas anastomoses e de infecções que invadem a traquéia. Ao redor das fístulas, forma-se excesso de tecido de granulação. ${ }^{34}$ Essas alterações foram observadas em ambos os grupos. A infecção é outro evento adverso que acompanha freqüentemente as cirurgias de traquéia, e pode causar a eliminação das próteses ou dos enxertos. ${ }^{21,35}$ Nos casos de infecção ou inflamação intensos, pode ocorrer disfagia por compressão extrínseca do esôfago. A perda ponderal nos animais do Grupo II pelo teste do Qui-quadrado foi maior, porém, não significativa e a ocorrência de infecção, estenose, fístulas e tecido de granulação foram observadas em ambos os grupos, possivelmente relacionados à técnica de sutura contínua, que, em algum grau, determina a isquemia.

Observou-se reepitelização e cicatrização nos animais submetidos aos transplantes autólogo e homólogo de traquéia e a evolução clínica dos animais de ambos os grupos evidencia a factibilidade e a aceitação de ambos os tipos de enxertos para tratamento de lesões traqueais.

O comportamento intra-operatório e a resposta à ventilação mecânica em ambos os enxertos foram similares, denotando resistência da anastomose e dos enxertos autólogo e homólogo à hiperpressão ventilatória. Ambos os enxertos são factíveis de suportar o estresse ventilatório relacionado à ventilação mecânica.

\section{CONCLUSÃO}

O comportamento intra-operatório, resposta à ventilação mecânica, a reepitelização e a cicatrização em coelhos submetidos a transplantes autólogo e homólogo de traquéia e a evolução clínica dos animais de ambos os grupos evidenciam a factibilidade e a aceitação de ambos os tipos de enxertos para tratamento de lesões traqueais, denotando a resistência da anastomose e dos enxertos autólogo e homólogo à hiperpressão ventilatória.

\section{AGRADECIMENTOS}

Ao Magnífico Reitor da Unifenas Prof. Dr. Edson Antonio Velano. À Prof- Ana Francisca Franco Oliveira.

\section{ABSTRACT}

Purpose: to study the cicatricial behavior and response to the intraoperative mechanical ventilation of the transplanted segment of the trachea in rabbits. Method: two groups were assessed: autologous (group I) and homologous transplant (group II) of the entire segment of the trachea in rabbits. Result: The Qui-square test showed no significant statistical difference between the studied groups. Regarding the time of survival, the Qui-square test $(\mathrm{p}<0.05)$ revealed that averages were considered statistically different (tc $=-3.60)$, and the survival of Group I was longer than Group II. The direct necroscopy and radiological assessment using the Kruskal-Wallis test ( $<<0.05$ ) showed no statistical difference between groups. Complications such as stenosis, formation of granulomas and fistulas were observed in both groups. Regarding the intraoperative behavior and response to the mechanical ventilation, the Kruskal-Wallis test ( $\mathrm{p}<0.05$ ) showed no significant statistical difference between Groups I and II. Both groups showed good resistance to the high pressure of the intraoperative mechanical ventilation. By using the Kruskal-Wallis test, no significant statistical difference was observed between groups concerning the anatomicopathological results. Group I had a higher prevalence of granuloma, bone metaplasia, necrosis in the region of the anastomosis, fibroplasia, graft reepithelialization, and polymorphonuclear infiltration. Conclusion: the reepitheliazation and early and late inflammatory 
reactions were observed in both groups, but animals with autologous transplant had a better clinical evolution and survival for a longer time than those which received homologous transplant.

Keywords: Trachea; Rabbits; Transplantation; Respiration, Artificial; Clinical Evolution; Survivorship.

\section{REFERÊNCIAS}

1. Acosta A, et al. Tracheal stenosis: the long and the short of it. Journal Pediatric Surgery 2000; 35:1612-16.

2. Backer C, et al. Intermediate-term results of the tracheal autograft for long segment congenital tracheal stenosis. Journal Pediatric Surgery. 2000;35:813-19.

3. Berdon W. Rings, slings, and other things: vascular compression of the infant trachea updated from the mid-century to the millennium - the legacy of Robert E. Gross and Edward B. D. Neuhauser. Radiology. 2000;216:624-32.

4. Matute JA, Romero R, Garcia-Casillas MA. Surgical approach to funnel-shaped congenital tracheal stenosis. Journal Pediatric Surgery 2001;36:320-3.

5. Burge D, Clements B, Warner J. Respiratory Medicine. London: Saunders; 1996.

6. Hansen T, Corbet A. Diseases of the airways. Philadelphia: Saunders; 1998.

7. Kon M, Hooff A. Cartilage tube formation by perichondrium. Plastic Reconstruction Surgery. 1983;72:791-5.

8. Krizek TJ, Kirchner JA. Tracheal reconstruction with an autogenous mucochondrial graft. Plastic Reconstruction Surgery 1972;50:123-30.

9. Lang F, Hurni M, Monnier P. Long segment congenital tracheal stenosis: treatment by slide-tracheoplasty. Journal Pediatric Surgery. 1999;34:1216-22.

10. Massone F. Anestesiologia Veterinária: Farmacologia e técnicas. Rio de Janeiro : Guanabara Koogan; 2003.

11. Moghissi K. Tracheal reconstruction with prosthesis of Marlex mesh and pericardium. Journal Thoracic Cardiovasculary Surgery. 1975;69:499-506.

12. Nelson RJ, White RA, Hirose FM. Neovascularity of a tracheal prosthesis tissue complex. Journal Thoracic Cardiovasculary Surgery .1983;86:800-8.

13. Bailey BJ, Kosoy J. Observations in the development of tracheal prostheses and tracheal transplantation. Laryngoscope. 1970;80:1553-65.

14. Taber RE, Tomatis L. Experimental and clinical utilization of a prosthesis for replacement of the trachea. Archives Surgery. 1958;77:576-83.

15. Jackson TL, et al. Tracheoplasty with pericardial patch for extensive tracheal stenosis in infants and children. Journal Thoracic Surgery. 1950;20:598-612.

16. Ferguson DJ, Wild JJ, Wangensteen $\mathrm{OH}$. Experimental resection of the trachea. Surgery. 1950;28:597-619.

17. Harrington $\mathrm{OB}$, et al. Circunferencial replacement of the trachea with Marlex mesh. American Surgery. 1962;28:217-23.

18. Vebakey ME, et al. The use of heavy Marlex mesh for tracheal reconstruction following resection for malignancy. Journal Thoracic Cardiovasculary Surgery. 1962;44:520-7.
19. Worman LW, Pemberton AH, Narodick BG. Tracheoplasty by means of a periosteal pedicle graft. Journal Thoracic Cardiovasculary Surgery 1964; 47: 572-6.

20. Rose KG, Sterhenn K, Wostron F. Tracheal allotransplantation in man. Lancet. 1979;1:433.

21. Otaviano AG, et al. Prótese traqueal. Revista Colaboração Brasileira Cirurgia. 1983;10:46-53.

22. Wykoff TW. A preliminary report on segmental tracheal prosthesis replacement in dogs. Laryngoscope. 1973;83:1072-7.

23. Nelson RJ, White RA, Hirose FM. Neovascularity of a tracheal prosthesis tissue complex. Journal Thoracic Cardiovasculary Surgery. 1983;86:800-8.

24. Alonso WA, Bridger GP, Bordley JE. Tracheal transplantation in dogs. Laryngoscope. 1972;82:204-9.

25. Tsang V, et al. Slide tracheoplasty for congenital funnel-sahped tracheal stenosis. Annual Thoracic Surgery. 1989;48;632-5.

26. Fialho SAG. Anestesiologia veterinária: Guia prático de anestesia para pequenos e grandes animais. São Paulo: Nobel; 1986.

27. Bailey B, Camp FA. Current concepts in reconstruction of the cervical trachea. American Surgery. 1969;35:153-65.

28. Beall JR, et al. Tracheal replacement with heavy Marlex mesh. Archives Surgery. 1962;84:390-6

29. Jatene FB, et al. Estudo experimental da dura-máter quando interposta em traquéia de cães. Ars Curandi. 1979;12:23-39.

30. Lang F, Hurni M, Monnier P. Long segment congenital tracheal stenosis: treatment by slide-tracheoplasty. Journal Pediatric Surgery. 1999;34:1216-22.

31. MontovaniI JC, Nakajima V. Alterações epiteliais e cartilaginosas em cirurgia traqueal: estudo experimental em cobaias. Revista Brasileira de Otorrinolaringologia. 2003;69:159-64.

32. Schneider A, et al. Estudo de viabilidade de aloenxerto de traquéia em ratos - o papel da omentoplastia na vigência de imunossupressão. Revista da Colaboração Brasileira em Cirurgia. 2000;27:189-92.

33. Neville WE, Bolanowisk PJP, Soltanzadeh H. Homograft replacement of the trachea using imunossuppression. Journal Thoracic Cardiovasculary Surgery. 1976;72:596-601.

34. Lane BP, Habicht GS, Jasper GS. Lymphocyte-epithelium interaction during rejection of nonisogeneic rat tracheal grafts. American Journal Pathology. 1977;86:71-7.

35. Filho C, Sanches J, Veríssimo F, Campos AD, Ellinguer F. Viability of comple tracheal segment transferred in the formo f a compound flap: Na experimental study in dogs. Laryngoscope. 114 (11): 1949-1952, November 2004. 